\title{
Construction of an exchange interface for the transmission of laboratory results: a case of the National Tuberculosis
} Center

\author{
Constant Joseph Koné ${ }^{1}$, Ndri Nda Anatole Mian ${ }^{1}$, Cataud Marius Guede ${ }^{1}$, Man-Koumba \\ Soumahoro ${ }^{1}$ \\ ${ }^{1}$ Pasteur Institute of Côte d'Ivoire, Epidemiology Unit
}

\begin{abstract}
Introduction

The transmission of test results by laboratories and their receipt by health facilities are common tasks in the processing of medical information. Managing the flow of information generated by these tasks remains a challenge for these centers. We describe a new system that will allow for electronic management of the transmission of results.
\end{abstract}

\section{Materials and methods}

The information system implemented is a client-server system consisting three main components: the server installed in the laboratory, the client distributed in the Anti-Tuberculosis Center and the communication channel represented by a Virtual Network. The exchange protocol is based on the HL7 standard that used messages of type ORU_R01.

Results

During the two months of implementation of this electronic result transmission system between the National Tuberculosis Reference Center in Abidjan and the Anti-Tuberculosis Center in Adzopé, which is about 110 kilometers away, twenty laboratory results were transmitted as soon as they left the laboratory, an improvement from the previous long turn-around-time of about 1 month. The minimalist interface and ease of use of the system have allowed it to be adopted by users.

\section{Discussion}

The use of the HL7 protocol for electronic notifications has proven its effectiveness in making transmissions of results instantaneous. Our system specifically addresses the problems related to efficient transmission of results; reduction of transmission time, information loss attributed to the use of paper, and transport costs incurred when transmitting results from remote sites. This system representing the 1rst version use a local codification that limits it to an interoperability with other environment that use a different code system. The use of a code system such as LOINC would allow full interoperability between different information systems.

Keywords: Information Systems, Tuberculosis, Health Facilities, HL7

Corresponding author: Constant Joseph Koné Email: koneconstant@pasteur.ci, koneconstant@yahoo.fr* DOI: 10.5210/ojphi.v11i3.10255 
This is an Open Access article. Authors own copyright of their articles appearing in the Online Journal of Public Health Informatics. Readers may copy articles without permission of the copyright owner(s), as long as the author and OJPHI are acknowledged in the copy and the copy is used for educational, not-for-profit purposes.

\section{Context and issues}

The transmission of test results from laboratories and their receipt by health facilities are common tasks in the processing of medical information. Unfortunately, they do not always satisfy patients and doctors, especially in terms of time [1]. The integration of information systems involved in the exchange of health data faces many problems due to the variability of the hardware, software, terminology, and nomenclatures used to code the information. Over the past two decades, standardized messaging protocols such as HL7 version 2 have helped to overcome the obstacles associated with syntactic interoperability. Its widespread adoption in health information systems makes it a standard of choice. Nevertheless, there are problems of interfacing between the different medical information systems, each system being specific, requires an adequate interface.

The transmission of results in electronic form from laboratories is an approach that goes hand in hand with computerized record systems. Several technologies have already proven their worth in this area. Direct Secure Messaging, a standardized protocol for the exchange of clinical messages and attachments, is a successful example of electronic transmission due to its flexibility and ease of implementation [2]. There are also commercial solutions such as Interfaceware's Iguana, which offers integrated systems for the exchange of health data [3].

The results submitted from laboratories in Côte d'Ivoire are mostly in the form of printed sheets of paper that must be sent to prescribing physicians. The latter can be subject to hazards such as transmission loss and delays, which have a negative impact on patient care. On the contrary, it has been shown that the transmission of results in the electronic form to the computerized patient record can greatly improve patient management [4].

Many systems have been developed for the electronic management of laboratory results. The vast majority of them suffer from their generic type design, a handicap due to the specificity of certain tasks and the limitations of the management of these laboratories. In this article, we describe a new system that, through its architecture and functionalities, will allow electronic management adapted to the transmission of results. Thus, its adoption by the laboratory will allow an essential saving of time in diagnosis, a rapid sharing of information and a more secure archiving.

\section{Material and method}

\section{System deployment environment: Case of Cote d'Ivoire}

The National Reference Center for Tuberculosis (NRCT) housed at the Pasteur Institute of Cote d'Ivoire is responsible for confirming the diagnosis of tuberculosis in samples from AntiTuberculosis Center (ATC) and health facilities (University Hospital Center, General Hospitals, health center) throughout the country. We are interested in ATCs, the main centers for tuberculosis management. There are 27 such centers across the country, while the NCRT is 
located in Abidjan in the south of the country (Figure 1). The remoteness and geographical accessibility of several ATCs can make it very difficult to transport samples and retrieve analytical results. Thus, as a solution, for many of them, the recovery of results is only done during the next sample transport, thus combining the recovery time of the result and the time of the next transmission. These time differences can sometimes reach several weeks for ACTs that are very far away. Electronic transmission of results would, therefore, be an asset inpatient care.

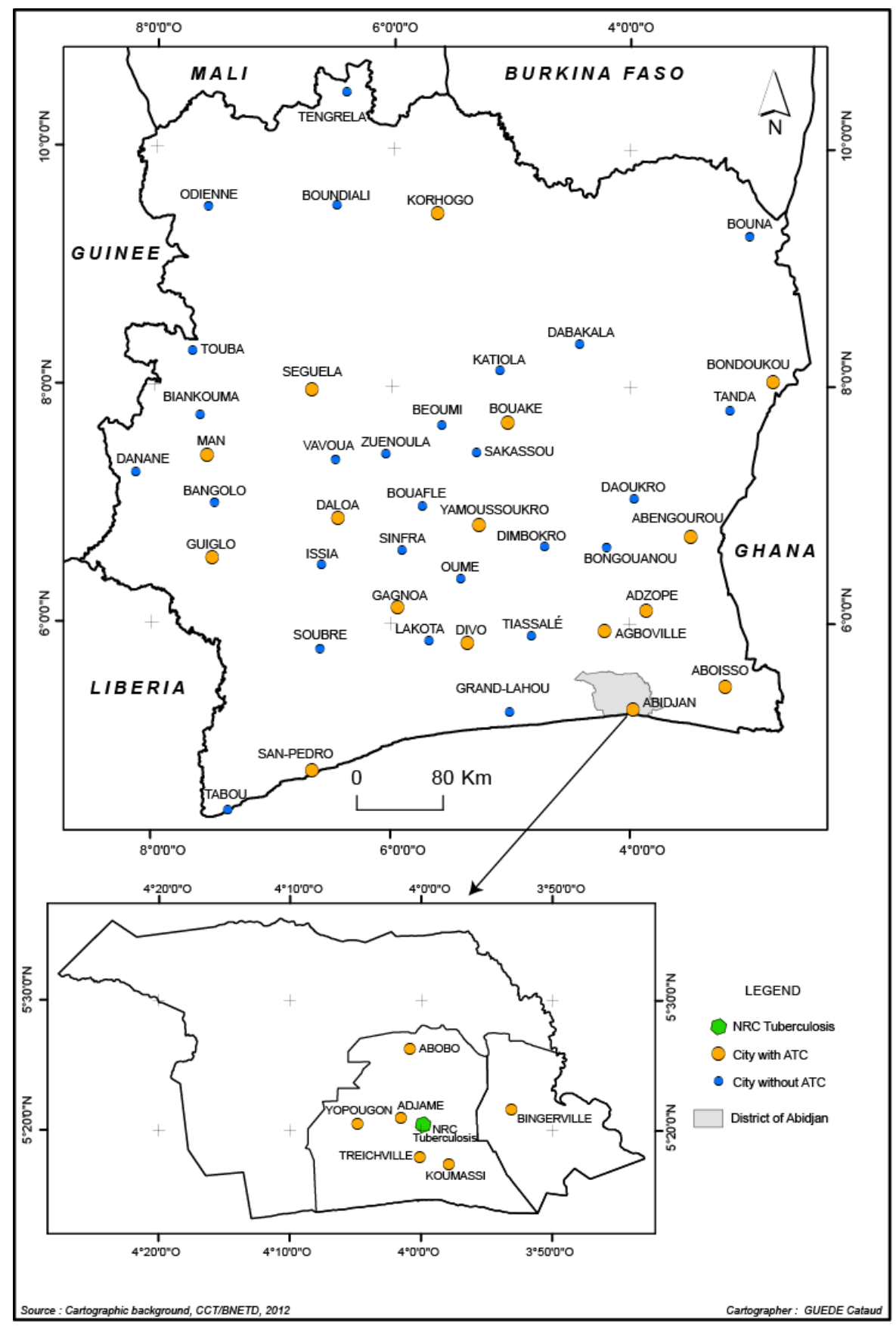

Figure 1: Distribution of ACTs in Côte d'Ivoire 


\section{System architecture and features}

The information system implemented is a client-server system including three main components: first, the server installed in the laboratory, then the client distributed in the ATCs and finally the communication channel represented by a Virtual Private Network (VPN). Figure 2 details the overall architecture of the data transmission information system.

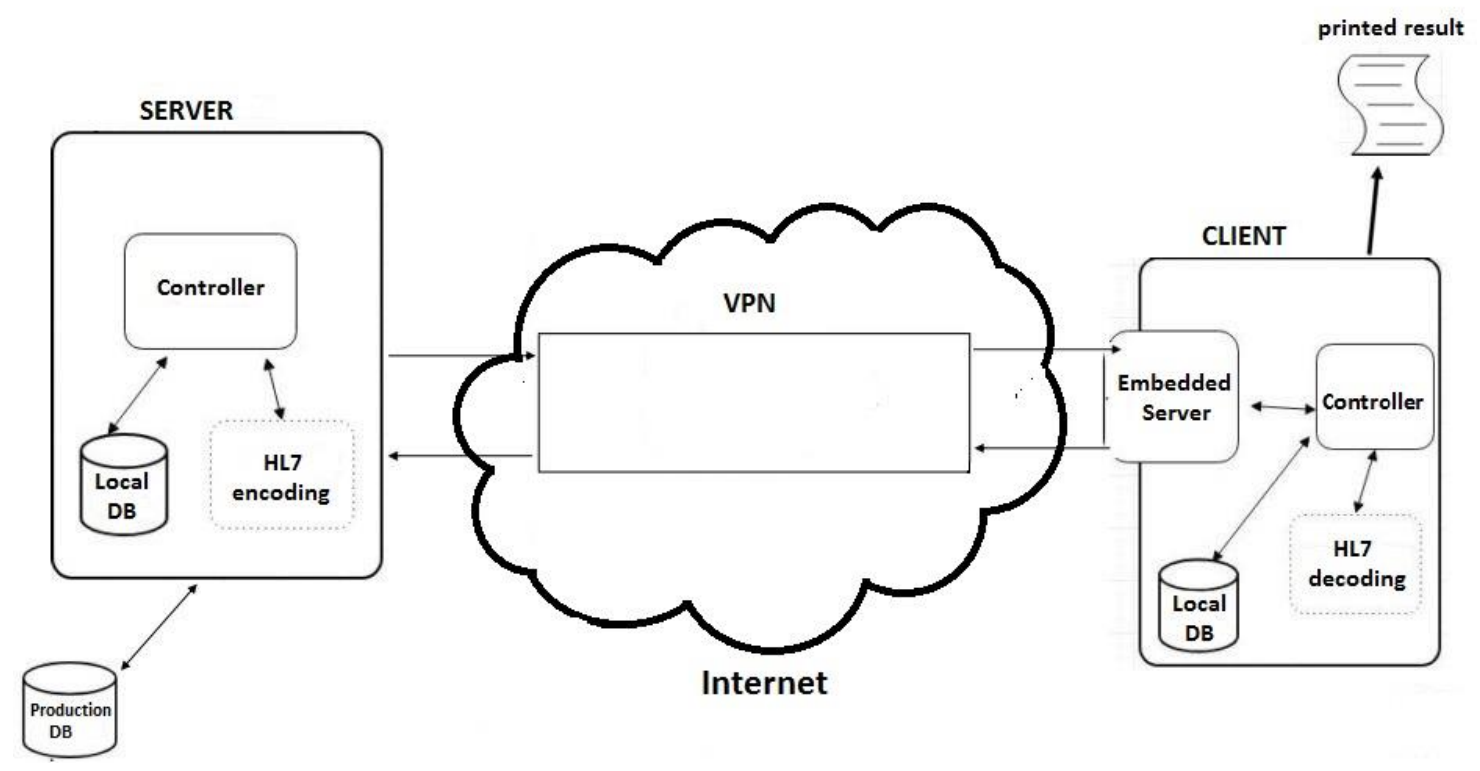

Figure 2: Information system architecture

\subsection{Server description}

The server software is installed at NRCT on a desktop computer. JAVA technology including JAVA standard edition is the main language used for the development of the system. Several other technologies are used in this system, including an embedded APACHE DERBY database that keeps track of all transactions, JASPER SOFT technology for PDF file generation, HL7 version 2.x standard and the HAPI library (HL7 Application Programing Interface) allowing its implementation in java [5-9].

The interface has been structured to be as simple as possible. Four actions on the graphical interface allow the transmission of a message:

1) The operator enters his identifier to unlock the graphical interface

2) He then enters the sample number in the relevant field. A query on the database of the existing laboratory information system retrieves all information related to this sample (sociodemographic information and biological results) [10].

3) Six laboratory tests (Microscopy, Gene expert, Line probe assay, Culture, Classical antibiotic susceptibility test, and extended antibiotic susceptibility test) can be generated by the server software. When the examination is actually performed, the server software displays it in its interface at the operator's request, otherwise, nothing is displayed. 
4) Finally, clicking on the Distribution button allows the result to be encoded in HL7 format and then sent over the Internet network through the VPN to the client software.

\subsection{Description of the customer}

The client software is also based on java technology, so it can be installed on any platform with a Java virtual machine. It implements an embedded database like the server to record all transactions. It also implements Jasper Soft technology to reconstruct the results received in PDF format. The client also has an embedded Jetty server that offers the possibility of having an open port waiting for incoming connections [11].

The graphical interface here is again minimalist, three actions are necessary to obtain the printed result.

1) The client software starts automatically when the operating system is launched and runs in the background. An icon marks its presence in the notification bar. When an incoming message is received, the client software displays a dialog box announcing the receipt of the new message. In terms of IT processes, when the message is received, it is decoded, the result of this processing generates a java object that is recorded in the embedded database. An acknowledgment of receipt is then generated and sent to the server sending the message.

2) By clicking on the notification, the client software is displayed, the interface is disabled, only the result can be viewed. The activation of the interface is done by entering its identifier in the relevant field. With the "print" button activated, it is possible to print the current result.

3) A table displays all the receipts of the day, a click on the "display" button of a line of the table, displays the result concerned and it is then possible to print it. Another table displays a summary of all transactions made and it is possible to display a previous result to print it.

\subsection{The communication channel}

A virtual private network is used between the server and the clients through the Internet network to secure data exchanges. We chose Hamachi's LogMeIn solution [12]. The star architecture is used here, it offers stricter control over network members and who is connected to whom. The server is connected to all workstations (clients), while the workstations are only connected to the server. This is a typical choice in a company, where workstations are only connected to servers. This architecture is ideal in the field of health where confidentiality is a necessity.

\section{Message structure}

The message structure is based on the HL7 standard version 2.5 [13]. The type of message used is ORU_R01, which refers to an unsolicited transmission of an observation message. In other words, the ORU message R01 is used to transmit the results of laboratory, clinical or other observations to other systems without the latter having requested it.

Twenty segments make up a message of type ORU_R01, some are optional, others mandatory. Five segments were used to implement the six laboratory results that the server software can 
transmit. These are the MSH (Message Header), PID (Patient Identification), OBR (Observation Request), OBX (Observation Segment), SPM (Specimen) segments. Figure 3 shows a detailed example of the structure of a microscopy message.

The HAPI library, implementation of the HL7 standard in JAVA, allowed the coding of messages. The HL7 over HTTP protocol was used for message transfer [9].

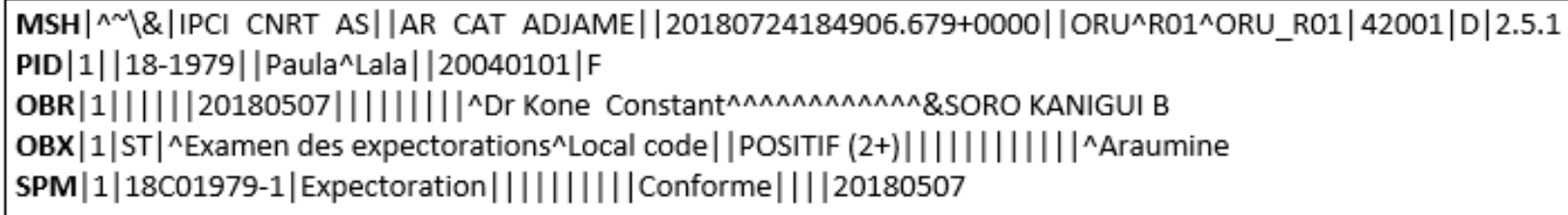

Figure 3: HL7 message of type ORU_R01

\section{On-site system installation, user training, and implementation}

The practical implementation of the system was carried out between the NRCT housed at the Institut Pasteur of Cote d'Ivoire, located in the city of Abidjan, and the ATC in the city of Adzopé, located about 110 kilometers from Abidjan.

We configured Hamachi's LogMeIn virtual private network on a laboratory workstation and the server was installed there. A password was created for each user. A training session was given on the use of the server at the NRCT to two laboratory technicians. The simplicity of use as we describe in server description, made it possible to conduct theoretical and practical training in half a day.

At the Adzopé ATC level, the VPN was configured, and the client software installed on a laptop computer. The medical secretary and the main doctor received theoretical and practical training.

The system was used for the transmission of results from 1 November 2018 to 31 December 2018. During these periods all test results were transmitted electronically.

\section{Results}

We only had to develop two interfaces for our project, one for the laboratory and one for the ATCs (Figure 4 and 5). Installation in other ATCs only required changing parameters such as IP address and interface label.

During the 2 months of implementation of this electronic result transmission system between the CNRT in Abidjan and the ATC in Adzopé, twenty laboratory results were transmitted. Three main advantages identified were:

1) At the time level: What is currently happening in the laboratory is that the transmission of the results is done manually, meaning that applicants must come and get their results from the NRCT, it can take a lot of time if the distance between CAT and the NRCT is great. Thus the electronic transmission of laboratory results undeniably saves time in the recovery of these results

2) At the financial level: This electronic system has resulted in substantial savings in fuel purchases and maintenance of the rolling stock needed to recover results. 
3) In terms of patient management: the results available in real-time have had a positive impact on improving the quality of consultations. The archiving of patient results and the possibility of regenerating these results later for consultation makes it more of a results directory and a patient data management tool.

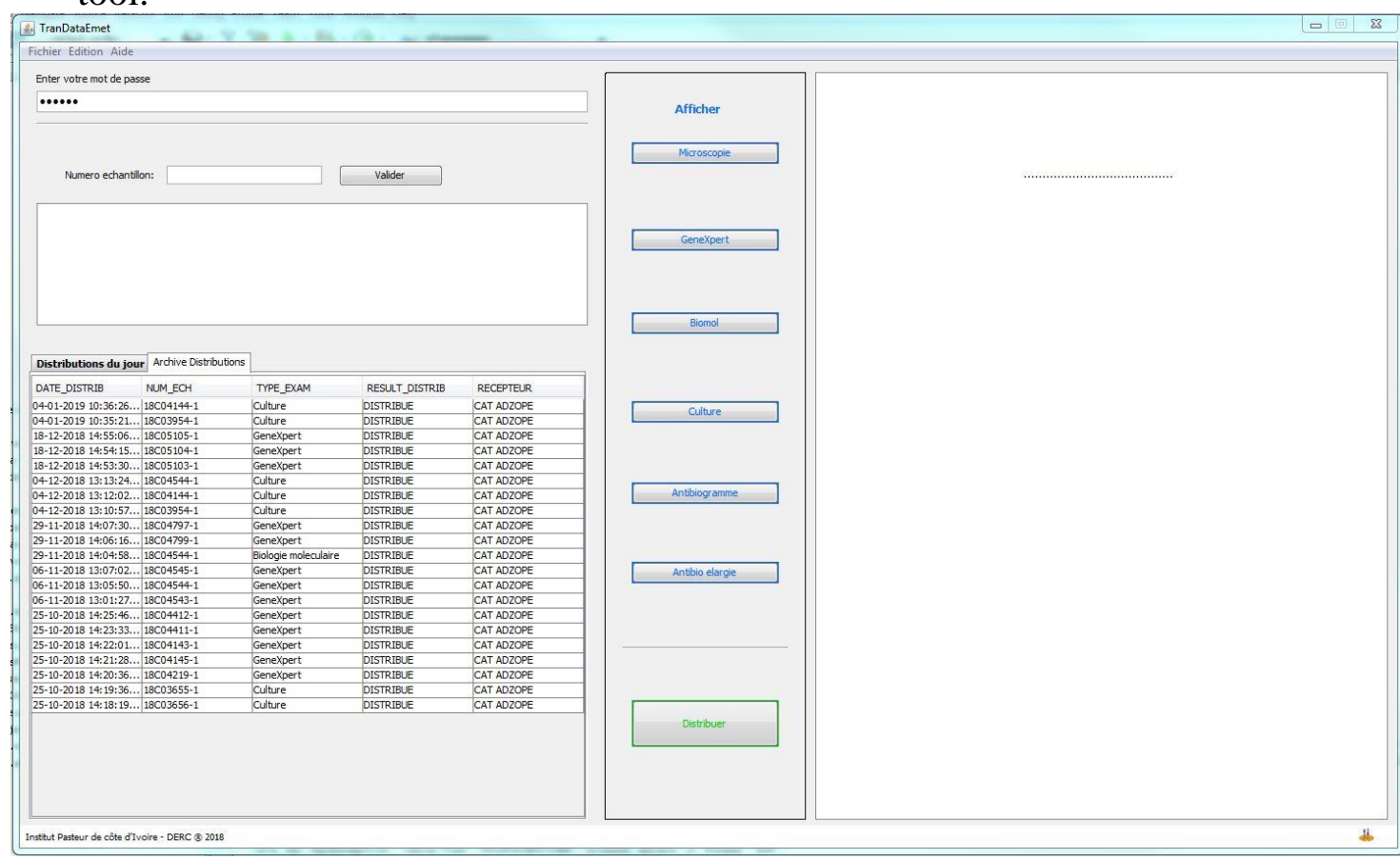

Figure 4: Server interface

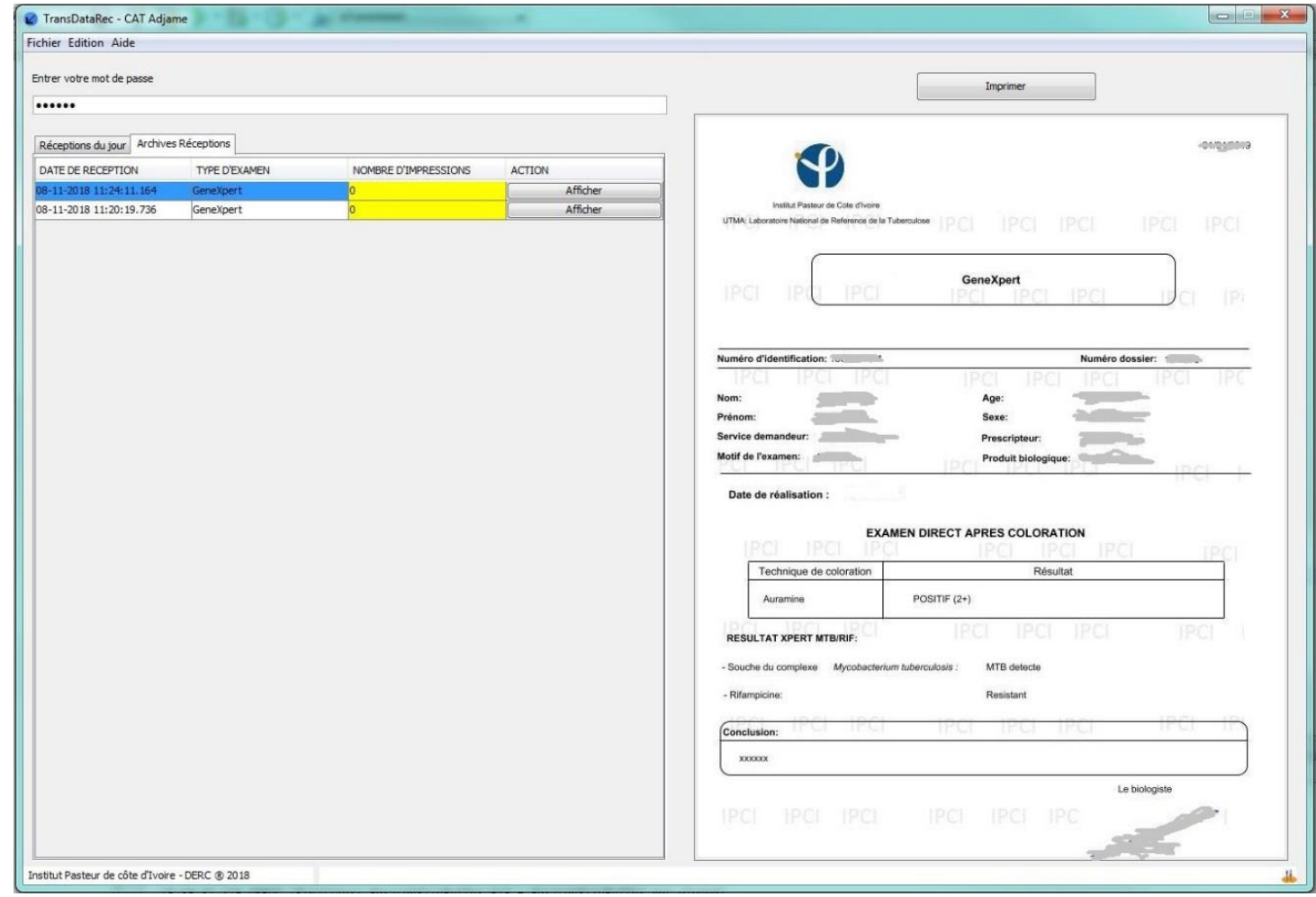

Figure 5: Client interface 


\section{Discussion}

The NRCT routine generates dozens of laboratory results per day for ATCs. We noted an accumulation of laboratory results not removed, probably due to the distance from the NRCT. The use of a system of electronic transmission of the results showed its effectiveness during the two months of implementation. Rajeev et al. showed that the use of electronic notification with the HL7 v 2.5.1 protocol is much faster and more complete. This observation is in line with our work, which allows laboratory results to be made available instantly [14].

Unlike DIRECT technology, which has the ability to transmit the results as an HL7 message that can be directly integrated into the medical file or as an attached PDF file, our system only transmits HL7 messages, which after decoding and saving are used to generate the results in PDF format in the receiving system.

Our approach seemed to us to be the best in the context of countries with limited resources where very little funding is allocated to the computerization of the health system. The purchase of licenses for commercial solutions for such projects can be a barrier in terms of their cost. This approach was also a factor in the adoption of the system. Users in the NRC or ATC were reluctant to have the data stored online (Cloud).

The minimalist interface, ease of learning and use are the positive elements reported by users at the laboratory and ATC level that have facilitated its adoption. This is in agreement with Kavuma, which showed that ease of learning is the most important element in the adoption of electronic health record systems [15].

\section{Limits}

Two types of limits were found:

The first during the test phase; a bug was found, it was manifested by an impossibility to transmit the Gene Expert type result. This was due to the software design defect, specifically an HL7 encoding error of the Gene Expert message. This problem had been controlled.

However, concerning the second type, our work has a limit in terms of semantic interoperability management. That can be explain that this application was primarily done for a specific institution. By so, the server is able to transmit messages to all information systems using the same protocol, but they cannot be interpreted by the receiving system. Indeed, the coding of the analyses uses a local code that would not be understood by a health information system that does not share the same code system.

In subsequent versions, we plan to use the LOINC (Logical Observation Identifier Names and Codes) coding system, which would then allow the results to be transmitted to other electronic systems such as computerized medical records using this terminology [16].

\section{Conclusion}

The implementation of this information system has shown satisfactory results in terms of financial savings, time savings, and improvement in the quality of care. Its minimalist interface, ease of learning and use have also enabled its adoption by different categories of users. 
The use of an international coding system for analysis coding is a requirement to address the challenges of semantic interoperability in order to deliver messages to other information systems such as Electronic Health Records.

The electronic transmission system for the transmission of results is an opportunity for countries with limited resources.

\section{References}

1. Poon EG, Gandhi TK, Sequist TD, Murff HJ, Karson AS, et al. 2004. "I wish I had seen this test result earlier!": Dissatisfaction with test result management systems in primary care. Arch Intern Med. 164(20), 2223-28. PubMed https://doi.org/10.1001/archinte.164.20.2223

2. Sujansky W, Wilson T. 2015. DIRECT secure messaging as a common transport layer for reporting structured and unstructured lab results to outpatient providers. J Biomed Inform. 54, 191-201. PubMed https://doi.org/10.1016/j.jbi.2015.03.001

3. « Iguana Integration Engine: Rapid, Reliable integration. Every time. -

INTERFACEWARE Inc ». [Online]. Available on:

https://www.interfaceware.com/iguana.html?gclid=Cj0KCQiA14TjBRD_ARIsAOCmO9 b2WqNvUSYXQRbvjNu-

Y5nM5mdWNczpxhUR5Xo73X3k_irpsqr70KUaAj1UEALw_wcB. [Accessed: 22-Apr2019].

4. Elder NC, McEwen TR, Flach J, Gallimore J, Pallerla H. 2010. The management of test results in primary care: does an electronic medical record make a difference. Fam Med. 42(5), 327-33. PubMed

5. « Java SE | Oracle Technology Network | Oracle ». [Online]. Available on: https://www.oracle.com/technetwork/java/javase/overview/index.html. [Accessed: 16Jan-2018].

6. «Apache Derby ». [Online]. Available on: https://db.apache.org/derby/. Accessed: 11Feb-2018].

7. «JasperReports® Library | Jaspersoft Community ». [Online]. Available on: https://community.jaspersoft.com/project/jasperreports-library. [Accessed: 16-Fan-2018].

8. « Health Level Seven International - Homepage | HL7 International ». [Online]. Available on: http://www.hl7.org/. [Accessed: 11-Feb-2018].

9. « HAPI - The Open Source HL7 API for Java ». [Online]. Available on: https://hapifhir.github.io/hapi-hl7v2/. [Accessed: 11-Feb-2018].

10. Koné CJ, Touré A, N'Dri MK, Nguessan R, Soumahoro MK. Development of an Electronic Information System for the Management of Laboratory Data of Tuberculosis and Atypical Mycobacteria at the Pasteur Institute in Côte d'Ivoire. J Health Man \& Info. 2019; 6(1): 1-6. 
11. jmcconnell, « Jetty - Servlet Engine and Http Server ». [Online]. Available on: https://www.eclipse.org/jetty/. [Accessed: 11-Feb-2018].

12. «LogMeIn Remote Access | Secure Remote Desktop Software ». [Online]. Available on: https://www.logmein.com/. [Accessed: 12-Feb-2018].

13. «HL7 Standards Product Brief - HL7 Version 2.5.1 Implementation Guide: Electronic Laboratory Reporting to Public Health, Release 1 (US Realm)| HL7 International ». [Online]. Available on:

http://www.hl7.org/implement/standards/product_brief.cfm?product_id=98. [Accessed: 12-Feb-2018].

14. Rajeev D, et al. 2011. Evaluation of HL7 v2.5.1 Electronic Case Reports Transmitted from a Healthcare Enterprise to Public Health. AMIA Annu Symp Proc. 2011, 1144-52. PubMed

15. Kavuma M. 2019. The Usability of Electronic Medical Record Systems Implemented in Sub-Saharan Africa: A Literature Review of the Evidence. JMIR Human Factors. 6(1), e9317. PubMed https://doi.org/10.2196/humanfactors.9317

16. Vreeman DJ, McDonald CJ, Huff SM. 2010. LOINC® - A Universal Catalog of Individual Clinical Observations and Uniform Representation of Enumerated Collections. Int J Funct Inform Personal Med. 3(4), 273-91. doi:https://doi.org/10.1504/IJFIPM.2010.040211. PubMed 